\title{
Testing the accuracy ratio of the Spatio-Temporal Epidemiological Modeler (STEM) through Ebola haemorrhagic fever outbreaks
}

\author{
F. BALDASSI ${ }^{1}$, F. D’AMICO ${ }^{1}$, M. CARESTIA ${ }^{1,2}$, O. CENCIARELLI $^{1}$, \\ S. MANCINELLI ${ }^{1,3}$, F. GILARDI ${ }^{1,3}$, A. MALIZIA ${ }^{1,2} *$ D. DI GIOVANNI ${ }^{1,2}$, \\ P. M. SOAVE ${ }^{1,4}$, C. BELLECCI ${ }^{1,2}$, P. GAUDIO ${ }^{1,2}$ AND L. PALOMBI ${ }^{1,3}$ \\ ${ }^{1}$ International Master Courses in Protection Against CBRNe events, Department of Industrial Engineering and \\ School of Medicine and Surgery, University of Rome Tor Vergata, Italy \\ ${ }^{2}$ Department Industrial Engineering, University of Rome Tor Vergata, Italy \\ ${ }^{3}$ Department of Biomedicine and Prevention, School of Medicine and Surgery, University of Rome Tor Vergata, Italy \\ ${ }^{4}$ Università Cattolica del Sacro Cuore, School of Medicine and Surgery, Rome, Italy
}

Received 28 July 2015; Final revision 2 October 2015; Accepted 2 November 2015; first published online 1 December 2015

\section{SUMMARY}

Mathematical modelling is an important tool for understanding the dynamics of the spread of infectious diseases, which could be the result of a natural outbreak or of the intentional release of pathogenic biological agents. Decision makers and policymakers responsible for strategies to contain disease, prevent epidemics and fight possible bioterrorism attacks, need accurate computational tools, based on mathematical modelling, for preventing or even managing these complex situations. In this article, we tested the validity, and demonstrate the reliability, of an open-source software, the Spatio-Temporal Epidemiological Modeler (STEM), designed to help scientists and public health officials to evaluate and create models of emerging infectious diseases, analysing three real cases of Ebola haemorrhagic fever (EHF) outbreaks: Uganda (2000), Gabon (2001) and Guinea (2014). We discuss the cases analysed through the simulation results obtained with STEM in order to demonstrate the capability of this software in helping decision makers plan interventions in case of biological emergencies.

Key words: Ebola virus, infectious disease, mathematical modelling, virology, viral haemorrhagic fever.

\section{INTRODUCTION}

Mathematical modelling has emerged as an important tool for gaining understanding of the dynamics of the spread of infectious diseases. The need for accurate models which describe the epidemic process are vital, as infectious disease outbreaks threaten national and international stability with their potential impact, especially on public health and the economy. Scientific

\footnotetext{
* Author for correspondence: Dr A. Malizia, Department Industrial Engineering, University of Rome Tor Vergata, Italy. (Email: malizia@ing.uniroma2.it)
}

literature proposes mathematical models for studying epidemics, outbreaks, and optimization of interventions [1]. These mathematical tools are extremely valuable also for risk analysis, although sometimes challenging to apply and decipher for non-specifically trained users. For this reason, the development of user-friendly software for the easy application and visualization of epidemic models is of great interest in the field of risk assessment and emergency management.

Infectious diseases have always been of great concern for humanity. The struggle with these agents appears to know no bounds, and the strong belief, diffused after the 1960s, that infectious diseases would be 
eradicated with the improvement in sanitation, antibiotics, vaccinations, medical science and medical care, is now wavering. The World Health Organization (WHO) states that infectious diseases are responsible for the death of over 17 million people a year [2] and billions of others are infected. Furthermore, infectious diseases are still among the major causes of mortality in developing countries [3]. The main causes for this situation is that the aetiological agents causing infectious diseases are able to adapt and evolve, therefore we can observe new emerging infectious diseases [4]; at present, bacteria which are able to swim in pure bleach or survive in a dose of penicillin are known. Moreover, some diseases which were believed to be eradicated, are re-emerging, sometimes after hundreds of years and/or even in a more virulent form [5].

Together with the threat of biological weapons, whose research is lately focused on microorganisms and lethal infectious diseases, we have great motivation to understand how infectious diseases spread in the population and, eventually, improve the countermeasures to control their diffusion. Mathematical epidemiology contributes to the understanding of the behaviour of infectious diseases, and allows predictions about their spread in the population. Detection, prevention, therapy and control programmes, all take advantage of mathematical modelling for their comparison, evaluation, optimization and implementation.

In order to prevent, or at least to reduce an epidemic outbreak (or to minimize an infection from spreading), there is the need of models that can accurately capture the main characteristics of the disease; in fact, being able to foresee disease propagation is vital to provide more effective response measures. These will be reliable only if model parameters are well estimated.

Epidemic modelling has three main aims. The first is to understand the spreading mechanism of the disease. For this, the essential part is a mathematical structure (equations give us threshold values and other constants which we use to describe the behaviour of the disease). The second aim is to predict the future course of the epidemic. The third is to understand how we may control the spread of the epidemic (education, immunization, isolation, and more, analysis of the international protocols to contain epidemics and pandemic events based on evidence). In order to make a reliable model and predictions to develop methods of control, we must be sure that our model describes the epidemic closely, i.e. it contains all its specific features. Therefore, it is important to validate models by investigating whether they fit the observed data.
In this article, we used the Spatio-Temporal Epidemiological Modeler [STEM (Eclipse Foundation; http://www.eclipse.org], a tool designed to help scientists and public health officials to create and use models of emerging infectious diseases. STEM uses mathematical models of diseases (based on differential equations) to simulate the development or space-time evolution of a disease. In particular, our challenge is to demonstrate the reliability of this tool by analysing three real cases of Ebola haemorrhagic fever (EHF) outbreaks: Uganda (2000) [6, 7], Gabon (2001) [8], and Guinea (2014) [9-11].

In particular, our approach consisted of an initial assessment of the validity of the software through a benchmark between simulations and epidemiological data from the past Uganda EHF outbreak (2000, SEBOV strain). Next, we applied the epidemiological data from another well known EHF outbreak (Zaire 1995, ZEBOV strain), and we further evaluated the software as tool to simulate the development and evolution of two real EHF outbreak, Gabon (2001) and the recent Guinea outbreak (2014). These two EHF outbreaks were due to ZEBOV strain.

\section{METHODS}

\section{STEM}

STEM software is Java-based, and is an open-source software project under the umbrella of the Eclipse foundation [12]. STEM allows users to create spatial and temporal models of emerging infectious diseases. It was designed to help developers, researchers and users to plug in their choice of models. It comes with a large number of existing compartment models, e.g. Susceptible/Infectious (SI), Susceptible/Infectious/Recovered (SIR) and Susceptible/ Exposed/Infectious/Recovered (SEIR) models pre-coded with both deterministic and stochastic engines, and a new model-building framework that allows users to rapidly extend existing models or to create entirely new models. These models could aid in understanding, and potentially preventing, the spread of a disease.

The STEM application has built-in Geographical Information System (GIS) data for almost every country in the world. It comes with data about country borders, populations, shared borders (neighbours), interstate highways, state highways, and airports. This data come from various public sources.

STEM treats the world as a graph within a modular and hierarchical modelling structure. From bottom to top, this structure has three basic levels: graphs, models, and scenarios. We refer to STEM tutorial 
(https://wiki.eclipse.org/Tutorials_for_Developers) for in-depth examination of all the software functions.

\section{SEIR epidemic model}

Among different mathematical epidemic models [1, 12-14], we fitted the data from Uganda (2000), Gabon (2001) and Guinea (2014) EHF outbreaks in a simple deterministic (continuous time) SEIR epidemic model [15]. Almost all existing literature [16-18] on Ebola epidemic prediction are based on this model.

Individuals can be part of one of the following epidemiological states:

Susceptible $(S)$ : healthy population at risk of contracting the disease.

Exposed (E): infected, but not yet infectious.

Infectious (I): infected and infecting others; capable of transmitting the disease.

Removed or Recovered (R): population that dies or recovers from the disease.

We assume the population is closed, that is, the effect of demographic changes (birth and natural death) is minimal during the course of the epidemic. The total population size $N$ is divided in four compartments: susceptible individuals at time $t, S(t)$, the exposed class $E$ $(t)$ with an average incubation period of $1 / \varepsilon$ days before progressing to the infectious class $I(t)$, and the removed [death $D(t)$ or recovered $R(t)$ ] class $R(t)$ (where without any ambiguity of notation, $R$ will henceforth be referred to as recovered class). Infectious individuals move to the $R$ class (death or recovered) at the per capita rate $1 / \gamma$. In the absence of treatment, the $R$ class is termed removed because individuals reaching it will never have the chance to rejoin the process $[14,18]$. The model flowchart is depicted in Figure 1.

$C(t)$ is not a compartment, but is needed to keep track of the cumulative number of Ebola cases from the onset of symptoms. $C(t)$ is not an epidemiological parameter, it is the sum of $I$ and $R$, thereby including all suspected and confirmed cases.

The epidemiological states and epidemiological features present in the SEIR compartment model are reported in Table 1.

\section{Differential equation model}

The above transmission process is modelled by the following system of nonlinear ordinary differential equations $[12,13]$ :

$\frac{\mathrm{d} \mathrm{S}(t)}{\mathrm{d} t}=-\beta S(t) I(t) / N$,

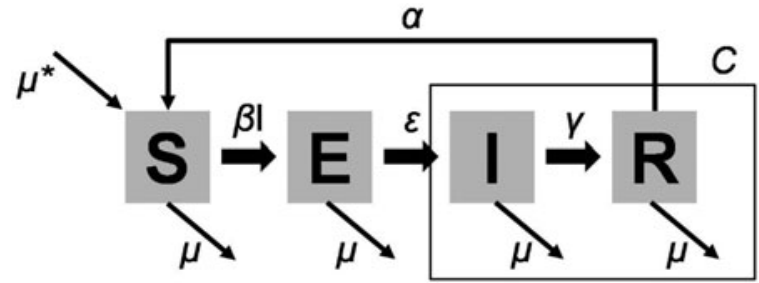

Fig. 1. SEIR compartment model. Epidemiological states: $S$, susceptible (healthy population at risk of contracting the disease); $E$, exposed (infected, but not yet infectious); $I$, infectious (infected and infecting others, capable of transmitting the disease); $R$, removed or recovered (population that dies or recovers from the disease); $C$, is not a compartment (includes $I$ and $R$ ); $\beta$, transmission rate; $\varepsilon$, incubation rate (per unit time); $\gamma$, recovery rate (per unit time); $1 / \gamma$, average recovery period; $\mu^{*}$, population birth rate; $\mu$, population death rate; $\alpha$, immunity loss rate.

Table 1. Epidemiological states and epidemiological features present in the SEIR compartment model. Explanation in extenso are reported in the text

\begin{tabular}{cl}
\hline \hline Epidemiological states & \\
$S$ & Susceptible \\
$E$ & Exposed \\
$I$ & Infectious \\
$R$ & Removed or recovered \\
$C$ & Suspected cases \\
Epidemiological features & \\
$N$ & Size of the population \\
$\varepsilon$ & Incubation rate \\
$\gamma$ & Recovery rate \\
$\delta$ & Infectious mortality rate \\
$\beta$ & Transmission rate \\
$1 / \gamma$ & Average recovery period \\
$1 / \varepsilon$ & Average incubation period \\
$\mu^{*}$ & Population birth rate \\
$\mu$ & Population death rate \\
$\alpha$ & Immunity loss rate \\
\hline \hline
\end{tabular}

$\frac{\mathrm{d} E(t)}{\mathrm{d} t}=-\beta S(t) I(t) / N-\varepsilon E(t)$,

$\frac{\mathrm{d} I(t)}{\mathrm{d} t}=\varepsilon E(t)-\gamma I(t)$,

$\frac{\mathrm{d} R(t)}{\mathrm{d} t}=\gamma I(t)$.

This model takes into consideration the number of people infected due to direct contact with an infected individual and the number of people infected due to indirect contact: $-\beta S(t) I(t) / N$. The individuals exhibit the symptoms of the disease and move on to the infectious stage. This is denoted by $\varepsilon E$, where $\varepsilon$ is the 
Table 2. Epidemiological features of three EHF outbreaks

\begin{tabular}{lll}
\hline \hline Epidemiological features & Value & Reference \\
\hline Uganda (2000) & & \\
Size of the population $(N)$ & 470000 & {$[16,27]$} \\
Number of index cases & 9 & {$[16]$} \\
Period considered & 18 Sept.-16 Oct. & {$[6,16,28]$} \\
Incubation rate $(\varepsilon)$ & $0 \cdot 083$ & {$[16]$} \\
Recovery rate $(\gamma)$ & $0 \cdot 1$ & {$[16]$} \\
Infectious mortality rate $(\delta)^{\mathrm{b}}$ & $0 \cdot 125$ & {$[16]$} \\
Transmission rate $(\beta)$ & $0 \cdot 505$ & {$[16]$} \\
Population density & 41 & {$[27]$} \\
Gabon $(2001)$ & & \\
Size of the population $(N)$ & $72 \cdot 461$ & $-^{\mathrm{a}}$ \\
Number of index cases & 1 & {$[15,18]$} \\
Period considered & 25 Oct.-16 Dec. & {$[8,29]$} \\
Incubation rate $(\varepsilon)$ & $0 \cdot 607$ & {$[18]$} \\
Recovery rate $(\gamma)$ & $0 \cdot 135$ & {$[18]$} \\
Infectious mortality rate $(\delta)^{\mathrm{b}}$ & $0 \cdot 135$ & {$[18]$} \\
Transmission rate $(\beta)$ & $0 \cdot 284$ & {$[18]$} \\
Population density & $5 \cdot 5$ & $-^{\mathrm{c}}$ \\
Guinea $(2014)$ & 1081675 & $-^{\mathrm{d}}$ \\
Size of the population $(N)$ & 1 & {$[15,18]$} \\
Number of index cases & 1 Dec.-22 Mar. & {$[9,26,30]$} \\
Period considered & $0 \cdot 607$ & {$[18]$} \\
Incubation rate $(\varepsilon)$ & $0 \cdot 135$ & {$[18]$} \\
Recovery rate $(\gamma)$ & $0 \cdot 135$ & {$[18]$} \\
Infectious mortality rate $(\delta)^{\mathrm{b}}$ & $0 \cdot 284$ & {$[18]$} \\
Transmission rate $(\beta)$ & 45 & $-\mathrm{d}$ \\
Population density & & \\
\hline \hline
\end{tabular}

${ }^{\mathrm{a}}$ Standard STEM population (2006).

${ }^{\mathrm{b}}$ Infectious mortality rate $(\delta)$ : shown only in the STEM compartment SEIR model.

${ }^{\mathrm{c}} \mathrm{http}: / /$ www.citypopulation.de/Gabon.html.

${ }^{\mathrm{d}}$ UN data - A world of information: Guinea (https://data.un.org/CountryProfile.aspx?crName=GUINEA).

per capita infectious rate. Then $1 / \varepsilon$ becomes the average time for a latent individual to become infectious. This will be denoted by $\gamma I$, where $\gamma$ is the per capita death rate. Then, $1 / \gamma$ becomes the average time it takes an individual to die once he/she has entered the infectious stage. As before, the number of dead and recovered individuals is assumed to be the same, since there has not been a case in which a person who survived Ebola contracts the disease again [14].

\section{Outbreak data}

The Ebola virus is a member of the family Filoviridae. The viruses in the family, called Filoviruses, cause severe haemorrhagic fever in humans and primates. Haemorrhagic fever is characterized by high fever, internal bleeding, hypotension and shock. There are five known strains of Ebola virus, each named according to the location where it was first recognized. These five strains are Ebola-Zaire (ZEBOV), Ebola-Sudan
(SEBOV), Ebola-Ivory Coast (CIEBOV), EbolaBundibugyo (BEBOV) and Ebola-Reston (REBOV) $[19,20]$.

The virus is spread through close contact and contaminated medical equipment. When a person comes into contact with, and becomes infected by the Ebola virus, the incubation period is $2-21$ days (average $8-10$ days). The onset of the illness is then abrupt and includes fever, headache, joint and muscle aches, sore throat and weakness, followed by diarrhoea, vomiting and stomach pain. This condition is called haemorrhagic fever and it is often fatal $[19,21]$. We analysed data from the three aforementioned EHF epidemics. A brief description of the outbreaks is given in the next sections.

\section{Uganda (2000)}

A total of 425 cases (case fatality rate $53 \%$ ) of Ebola were identified in three districts of Uganda: Gulu, Masindi and Mbara [22-25]. The onset of symptoms 
(a)

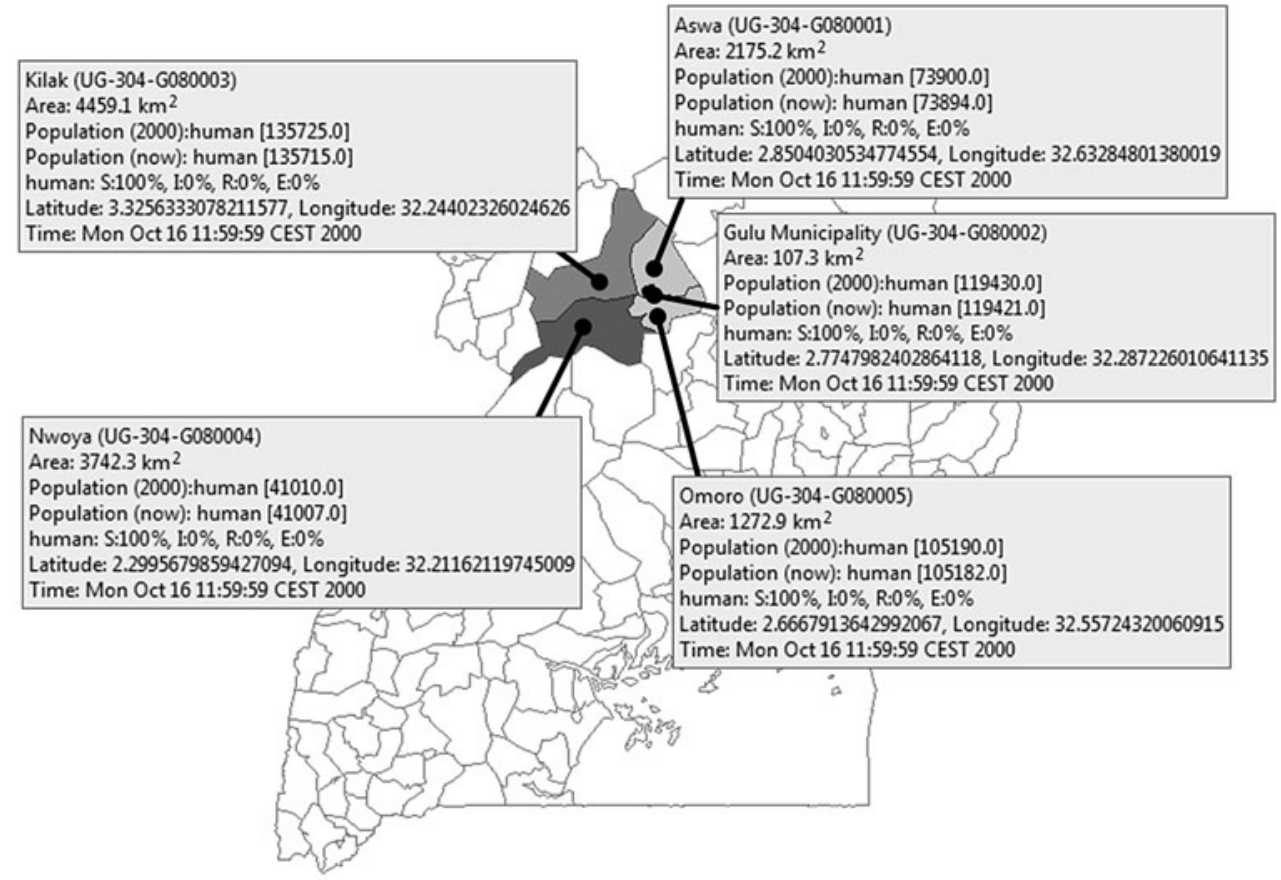

(b)

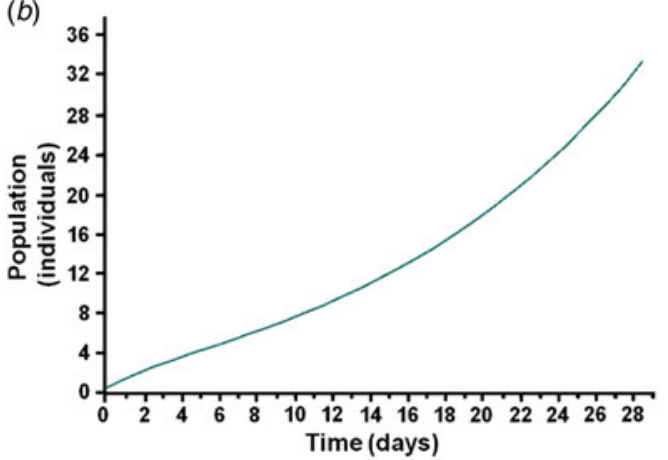

(c)

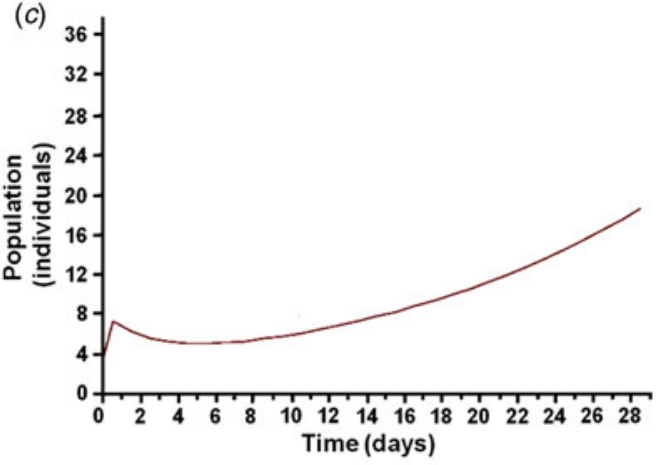

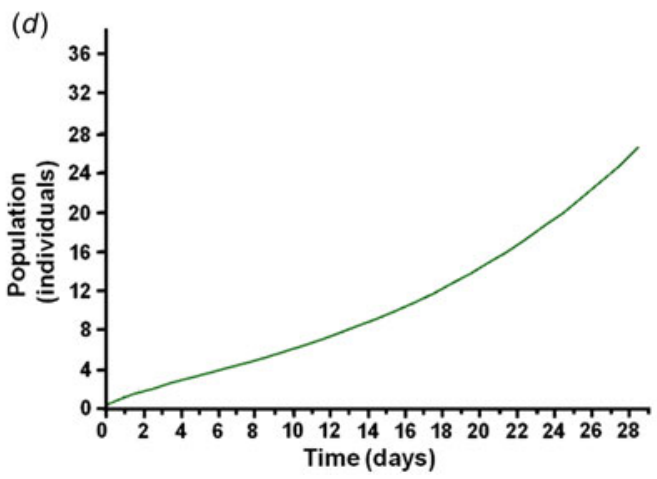

Fig. 2. Results of STEM simulation of the Uganda scenario. (a) Map view of the geographical distribution of the disease deaths; in particular, in the square with a red border the main information is reported: i.e. the name of the region considered, the area extension in $\mathrm{km}^{2}$ and the coordinates of the region, the population numbers before the disease occurred, the population numbers after the period considered that the disease occurred and the end time of the period considered. (b) Disease deaths, (D)t. (c) Infected people, (I)t. (d) Recovered people, $(R) t$. Time in days.

of the first reported case was on 30 August, but the cause was not identified as Ebola until 15 October by the National Institute of Virology in Johannesburg (South Africa).
Most of the 425 presumptive cases (confirmed and clinically diagnosed) occurred in the district of Gulu (470 000 inhabitants [6]). For this reason, we fitted our model with Gulu district data. 
Table 3. Results of the three EHF outbreaks simulation. Real and simulated data are reported together with a percentage estimation of the accuracy

\begin{tabular}{llll}
\hline \hline EHF outbreak & $\begin{array}{l}\text { Real } \\
\text { data }\end{array}$ & $\begin{array}{l}\text { Simulated } \\
\text { data }\end{array}$ & $\begin{array}{l}\text { Accuracy } \\
(\%)\end{array}$ \\
\hline $\begin{array}{l}\text { Uganda (2000) } \\
\quad \text { Deaths }\end{array}$ & 35 & 36 & $97 \cdot 2$ \\
$\quad \begin{array}{l}\text { Suspected } \\
\text { cases }\end{array}$ & 71 & 77 & $92 \cdot 2$ \\
$\begin{array}{l}\text { Gabon (2001) } \\
\quad \begin{array}{l}\text { Deaths } \\
\text { Suspected }\end{array}\end{array}$ & 12 & 11 & \\
$\begin{array}{l}\text { cases } \\
\text { Guinea (2014) }\end{array}$ & 15 & 16 & $91 \cdot 7$ \\
$\begin{array}{l}\text { Deaths } \\
\text { Suspected } \\
\text { cases }\end{array}$ & 29 & 29 & $93 \cdot 7$ \\
\hline \hline
\end{tabular}

Gabon (2001)

The first index case was probably infected during a hunt near Mendemba village, on 21 October 2001, and the last index case was infected near Grand Itoumbi village on 23 February 2002. During this epidemic, most secondary cases were related to community-based transmission. All cases observed in the Ivindo district were linked to two imported cases from La Zadié, which were admitted to Makokou Regional Hospital. Two healthcare workers were infected: one in Mékambo Health Centre (La Zadié district, Gabon), and one in Makokou Regional Hospital (Ivindo district, Gabon) [8].

Guinea (2014)

On 23 March 2014, the WHO issued its first public announcement on a new outbreak of Ebola virus disease, which began in December 2013 in the Republic of Guinea [9-11, 26]. The initial source of the outbreak appears to be the village of Meliandou in Gueckedou Prefecture, and the index case was a 2-year-old child who died on 6 December 2013. From the start of the outbreak to 22 March 2014, a total of 49 cases including 29 deaths (case fatality ratio $59 \%$ ) were reported $[9,10,11,26]$.

\section{Epidemiological features of the three EHF outbreaks}

See Table 2 for epidemiological features of the three EHF outbreaks.

\section{Assumptions}

To run the simulations, we made the following assumptions for the epidemics:
(1) The entire population was initially considered susceptible, and as a result, at the beginning of the epidemic $N(t)=S(t)$.

(2) The population considered is a constant population during the simulation of all outbreaks. This means that there are no deaths due to outside factors and the number of births that occurred are so small that we overlook them. As a result, we can safely ignore the $\mu^{*}$ and $\mu$ parameters.

(3) For each outbreak simulation the SEIR epidemic model was respectively initialized with the number of index cases indicated in Table 2 (see 'Number of index cases').

(4) All observed EHF cases (deaths and suspected cases) were assumed to be related to human-to-human transmission.

(5) For each outbreak the period of time evaluated, indicated in Table 2 as 'Period considered', go from the onset of outbreak to the initial control interventions (hospitalization, disease control measures, quarantine, etc.). In this way we can study the real distribution of the outbreak, without external interventions.

(6) The suspected cases $(C)$ are the combination of $I$ and $R(D)$ individuals.

(7) To simulate the Uganda outbreak we assumed that the index cases were distributed uniformly in all Gulu districts; this is because the literature from where we obtain the epidemiological data of the EHF Uganda outbreak [16] does not detail the real geographical distribution of these index cases.

(8) For simulating Gabon and Guinea outbreaks we used the epidemiological data of the Congo outbreak (1995). This allows evaluation of the software as tool for simulating the development and evolution of two real EHF outbreak using the epidemiological data from another EHF outbreak, but caused by the same strain (ZEBOV).

\section{RESULTS}

We use the parameters in Table 2 to simulate the EHF outbreaks in Uganda (2000), Gabon (2001) and Guinea (2014) through STEM software.

According to the reference literature from WHO [28], the EHF outbreak in Uganda in 2000, produced 71 suspected cases including 35 deaths in Gulu district, northern Uganda in the reference period between 18 September and 16 October in 2000. We fit the 
(a)

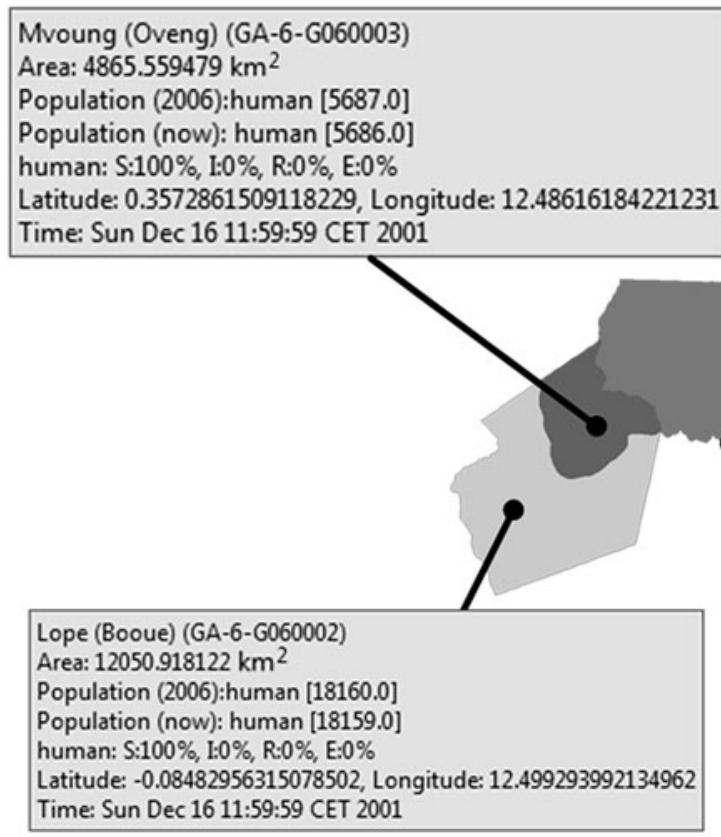

Zadie (Mekambo) (GA-6-G060004) Area: $10602.372273 \mathrm{~km}^{2}$

Population (2006): human [14746.0]

Population (now): human [14739.0]

human: $\mathrm{S}: 100 \%, \mathrm{I}: 0 \%, \mathrm{R}: 0 \%$, $\mathrm{E}: 0 \%$

Latitude: 1.0182710303519595 , Longitude: 14.08390674946827

Time: Sun Dec 16 11:59:59 CET 2001
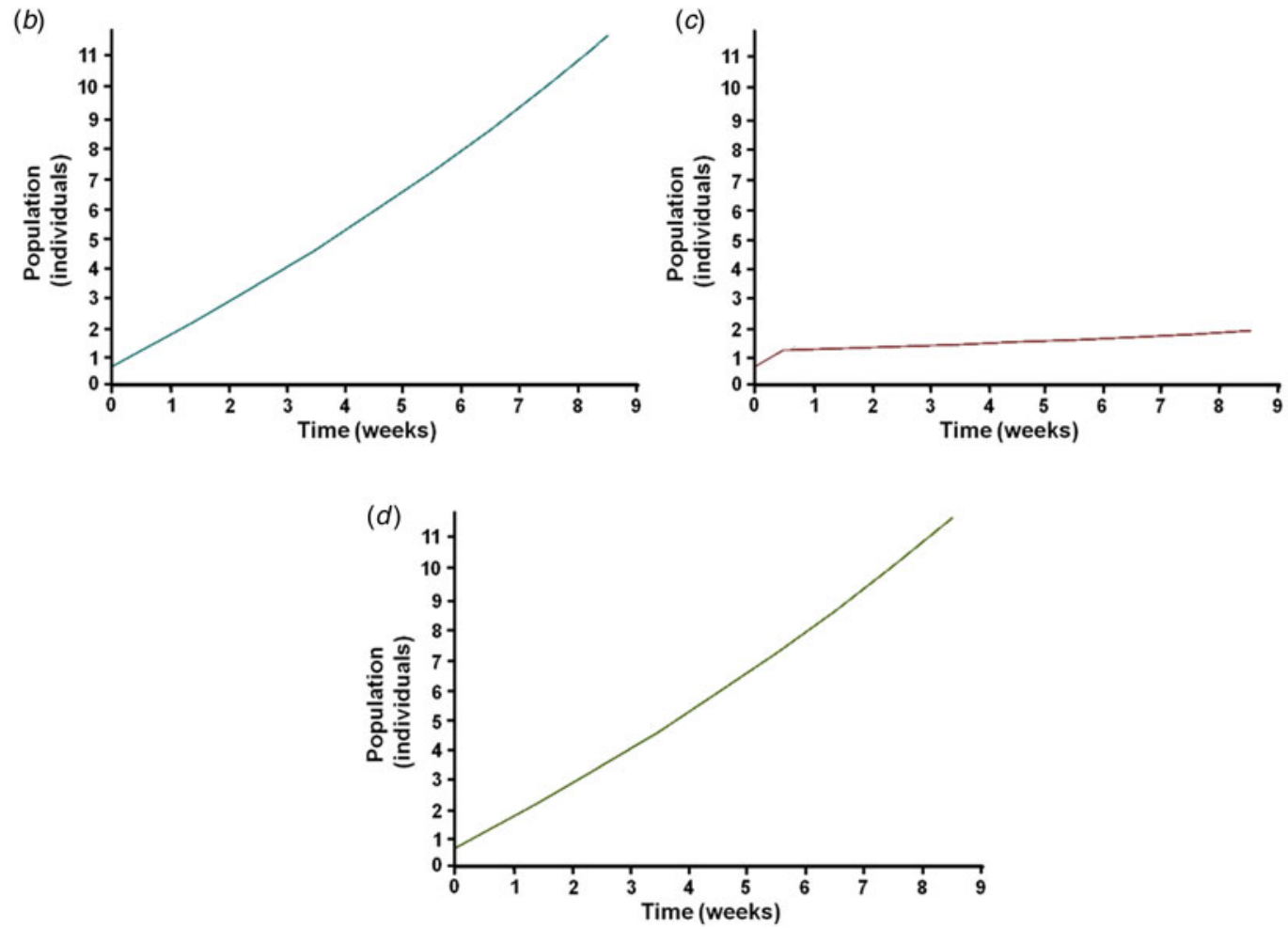

Fig. 3. Results of STEM simulation of the Gabon scenario. (a) Map view of the geographical distribution of the disease deaths; in particular, in the square with the red border the main information is reported: i.e. the name of the region considered, the area extension in $\mathrm{km}^{2}$ and the coordinates of the region, the population numbers before the disease occurred, the population numbers after the period considered that the disease occurred and the end time of the period considered. (b) Disease deaths, (D)t. (c) Infected people, $(I) t$. (d) Recovered people, $(R) t$. Time in weeks. 
(a)
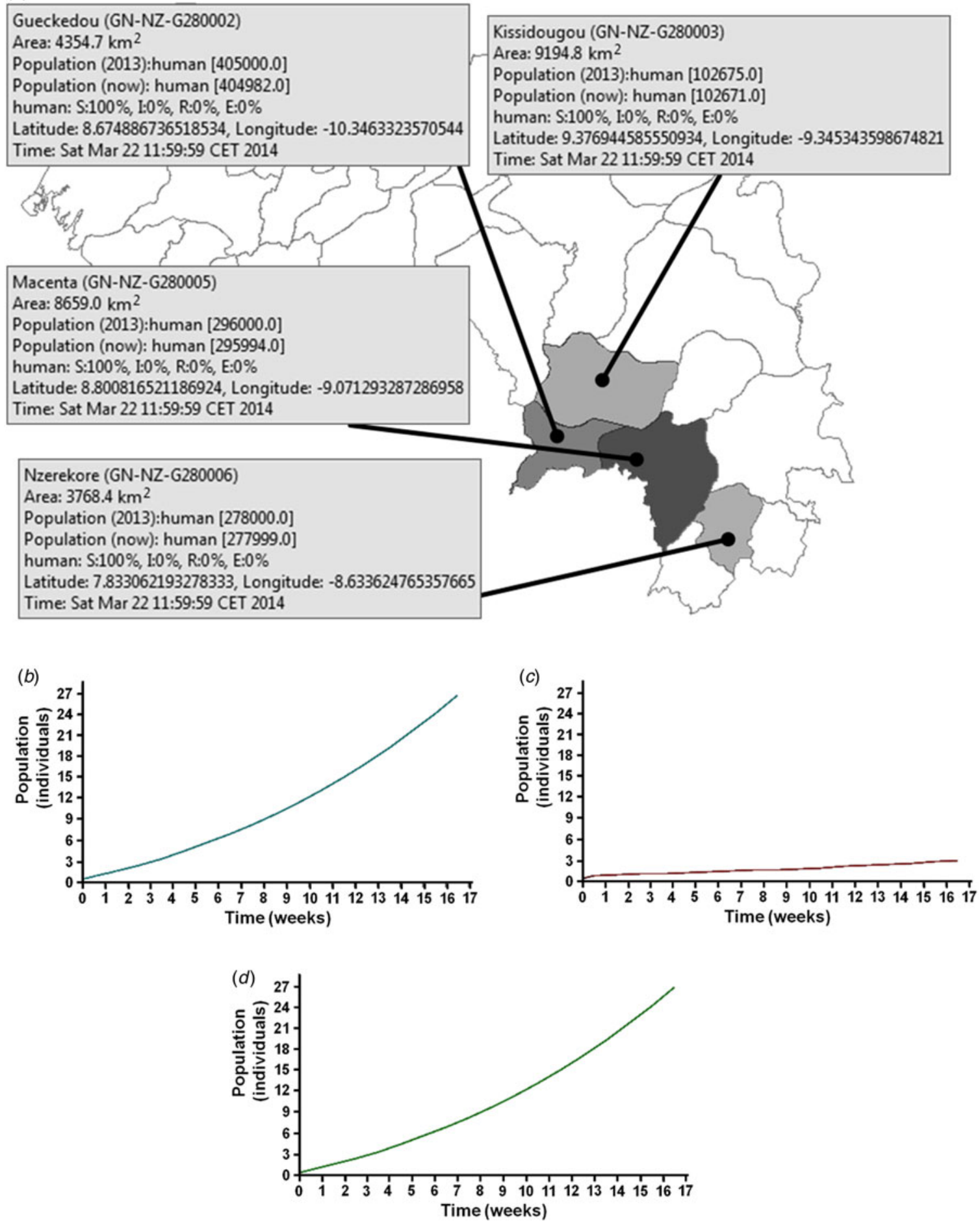

Fig. 4. Results of STEM simulation of the Guinea scenario. (a) Map view of the geographical distribution of the disease deaths; in particular, in the square with the red border the main information is reported: the name of the region considered, the area extension in $\mathrm{km}^{2}$ and the coordinates of the region, the population numbers before the disease occurred, the population numbers after the period considered that the disease occurred and the end time of the period considered. (b) Disease deaths, (D)t. (c) Infected people, $(I) t$. (d) Recovered people, $(R) t$. Time in weeks. 
epidemiological features in Table 2 in STEM and the results of the simulation are given in Figure 2 and Table 3.

In the EHF outbreak in Gabon (2001), according to the WHO [29], in the considered period of 25 October-16 December 2001, the Gabon Ministry of Health had reported 15 suspected cases (two laboratory confirmed) including 12 deaths in Ogooué Ivindo province in the northeastern part of the country. The simulated data obtained with STEM for this scenario are reported in Figure 3 and Table 3.

Finally, in the last EHF outbreak and according to WHO reports [26], in the considered period of 1 December 2013-22 March 2014, the Ministry of Health $(\mathrm{MoH})$ of Guinea had notified a total of 49 cases including 29 deaths in Guekedou, Macenta, Nzerekore and Kissidougou districts in the east part of the country. We fit the epidemiological features in STEM software and the results of the simulation are indicated in Figure 4 and Table 3.

\section{DISCUSSION}

We simulated three different Ebola outbreaks using STEM and the results showed an elevated accuracy within the real and simulated data. This means that the SEIR compartmental model and STEM software could explain the epidemiological dynamics of Ebola virus (ZEBOV and SEBOV). Nevertheless, simplifying assumptions of the model implies that the results of this study need to be interpreted with caution.

In the EHF Uganda outbreak simulation the results obtained with STEM matched $>90 \%$ with the real data and this outcome gives us the reasonable certainty that it can represent the epidemiological data obtained from the literature.

Other significant data come from the study performed with the epidemiological features of the EHF Zaire outbreak (1995) applied to the Gabon and Guinea EHF epidemics caused by the same strain of Ebola virus (ZEBOV). We arrange the epidemiological data from the Zaire outbreak in order to understand the behaviour of the disease in the cases of Gabon and Guinea. As can be seen in Table 3, the results are significant and considerable. In fact, the simulated data match the case of the Gabon outbreak by $>91 \%$ and match the recent case of Guinea by $87-100 \%$. However, in the last Ebola case we emphasize that the full-length genome sequencing and phylogenetic analysis showed that the ZEBOV from Guinea forms a separate clade in relation to the known EBOV strains from the Zaire and Gabon outbreaks.

Overall, STEM software overestimates the real data, with few exceptions. In this regard, it should be emphasized that the period considered in the STEM simulations referred only to the onset of the disease until the initial interventions. We decided to proceed in this way in order to understand if STEM could characterize the natural distribution of the outbreak without external interventions, such as medical countermeasures or quarantine.

\section{CONCLUSIONS}

The outcomes of this study suggest that if the epidemiological features of a specific contagious disease are already known, STEM software could be a useful tool for understanding, with a high level of accuracy, how the outbreak will spread. As a result, this opensource software could became, with some limitations, an additional powerful weapon to evaluate the dynamics of the spread of infectious diseases whether they are the result of a natural epidemic or nonconventional human activities.

\section{ACKNOWLEDGMENTS}

The authors thank the International Master Courses in Protection Against CBRNe events for support both technically and economically in the realization of this work (www.mastercbrn.com).

\section{DECLARATION OF INTEREST}

None.

\section{REFERENCES}

1. Bachinsky AG, Nizolenko LP. A Universal Model for Predicting Dynamics of the Epidemics Caused by Special Pathogens. BioMed Research International. Published online: 7 July 2013. doi:10.1155/2013/467078.

2. World Health Organization. The world health report 1996: fighting disease, fostering development. Geneva: World Health Organization, 1996, pp. 137.

3. World Health Organization. The top ten causes of death. (http://www.who.int/mediacentre/factsheets/fs310/en/). Accessed 26 May 2015.

4. Morse SS. Factors in the emergence of infectious diseases. Emerging Infectious Diseases 1995; 1: 7-5.

5. Morens DM, Folkers GK, Fauci AS. The challenge of emerging and re-emerging infectious diseases. Nature 2004; 430: 242-249. 
6. Okware SI, et al. An outbreak of Ebola in Uganda. Tropical Medicine and International Health 2002; 7: 1068-1075.

7. World Health Organization. Outbreak of Ebola hemorrhagic fever, Uganda, August 2000-January 2001. Weekly Epidemiological Record 2001; 76: 41-46.

8. World Health Organization. Outbreak(s) of Ebola hemorrhagic fever, Congo and Gabon, October 2001-July 2002. Weekly Epidemiological Record 2003; 78: 217-228.

9. Baize S, et al. Emergence of Zaire Ebola virus disease in Guinea - preliminary report. New England Journal of Medicine 2014; 371: 1418-1425.

10. Gatherer D. The 2014 Ebola virus disease outbreak in West Africa. Journal of General Virology 2014; 95: 1619-1624.

11. Cenciarelli O, et al. Ebola virus disease 2013-2014 outbreak in West Africa: an analysis of the epidemic spread and response. International Journal of Microbiology. Published online: 17 March 2015. doi:10.1155/2015/ 769121.

12. Eclipse Foundation. (https://www.eclipse.org/org/). Accessed 26 May 2015.

13. Lavine JS, Poss M, Grenfell BT. Directly transmitted viral diseases: modeling the dynamics of transmission. Trends in Microbiology 2008; 16: 165-172.

14. Ndanguza D, Tchuenche JM, Haario H. Statistical data analysis of the 1995 Ebola outbreak in the Democratic Republic of Congo. Afrika Matematika 2013; 24: 55-64.

15. Hethcote HW. The Mathematics of Infectious Diseases. SIAM Review 2000; 42: 599-653.

16. Chowell G, et al. The basic reproductive number of Ebola and the effects of public health measures: the case of Congo and Uganda. Journal of Theoretical Biology 2004; 229: 119-126.

17. Legrand $\mathbf{J}$, et al. Understanding the dynamics of Ebola epidemics. Epidemiology \& Infection 2007; 135: 610-621.

18. Lekone P, Finkenstädt B. Statistical inference in a stochastic epidemic SEIR model with control intervention: Ebola as a case study. Biometrics 2006; 62: 1170-1177.

19. Sanchez A. Filoviridae: Marburg and Ebola viruses. In Knipe DM, Howley PM, eds. Fields Virology, 4th edn.
Philadelphia: Lippincott-Raven Publishers, 2001, pp. 1279-1304.

20. Cenciarelli O, et al. Biological emergency management: the case of Ebola 2014 and the air transportation involvement. Journal of Microbial and Biochemical Technology 2014; 6: 247-253.

21. World Health Organization. Ebola and Marburg virus disease epidemics: preparedness, alert, control, and evaluation. INTERIM version 1.2 (http://apps.who.int/ iris/bitstream/10665/130160/1/WHO_HSE_PED_CED_ 2014.05_eng.pdf). Accessed 26 May 2015.

22. Centers for Disease Control and Prevention. Ebola hemorrhagic fever information packet (www.cdc.gov/ncidod/ dvrd/spb/mnpages/dispages/Fact_Sheets/Ebola_Fact_ Booklet.pdf). Accessed 26 May 2015.

23. Muyembe-Tamfum JJ, et al. Ebola virus outbreaks in Africa: past and present. Onderstepoort Journal of Veterinary Research 2012: 79: 6-13.

24. Centers for Disease Control and Prevention. Outbreak of Ebola hemorrhagic fever Uganda, August 2000January 2001. Morbidity and Mortality Weekly Report 2001; 50: 73-77.

25. Francesconi $\mathbf{P}$, et al. Ebola hemorrhagic fever transmission and risk factors of contacts, Uganda. Emerging Infectious Diseases 2003; 9: 1430-1437.

26. World Health Organization. Ebola virus disease, West Africa. Weekly Epidemiological Record 2014; 89: 205-220.

27. Althaus CL. Estimating the reproduction number of Ebola Virus (EBOV) during the 2014 outbreak in West Africa. PLOS Currents Outbreaks. Published online: 2 September 2014. doi:10.1371/currents.outbreaks. 91afb5e0f279e7f29e7056095255b288.

28. World Health Organization. Ebola hemorrhagic fever in Uganda (http://www.who.int/csr/don/2000_10_16/en/). Accessed 26 May 2015.

29. World Health Organization. Ebola hemorrhagic fever in Gabon - Update 4 (http://www.who.int/csr/don/2001_ 12_16/en/). Accessed 26 May 2015.

30. World Health Organization. Ebola virus disease in Guinea (http://www.who.int/csr/don/2014_03_23_ ebola/en). Accessed 26 May 2015. 